\title{
RSUME regulates tumorigenesis and metastasis in pancreatic neuroendocrine tumors
}

Yonghe Wu1,6, Lucas Tedesco2 ${ }^{1}$, Kristin Lucia1, Anna M. Schlitter ${ }^{3}$, Irene Esposito ${ }^{3,7}$, Christoph J.

Auernhammer ${ }^{4}$, Marily Theodoropoulou ${ }^{1}$, Eduardo Arzt ${ }^{2,5}$, Ulrich Renner ${ }^{1}$, Günter K. Stalla ${ }^{1}$

1Department of Clinical Neuroendocrinology, Max Planck Institute of Psychiatry, Munich, Germany; ${ }^{2}$ Instituto de Investigación en Biomedicina de Buenos Aires (IBioBA) - CONICET - Partner Institute of the Max Planck Society, Buenos Aires, Argentina; ${ }^{3}$ Institute of Pathology, Technical

University of Munich, Munich, Germany; ${ }^{4}$ Department of Internal Medicine II, University-Hospital Campus Grosshadern, Interdisciplinary Center of Neuroendocrine Tumours of the GastroEnteroPancreatic System (GEPNET-KUM), Ludwig-Maximilians-University of Munich, Munich, Germany;

${ }^{5}$ Departamento de Fisiología y Biología Molecular y Celular, Facultad de Ciencias Exactas y Naturales, Universidad de Buenos Aires, Buenos Aires,

Argentina; ${ }^{6}$ Current address: German Cancer Research Center, Heidelberg, Germany; ${ }^{7}$ Current address: Institute of Pathology, University of

Düsseldorf, Düsseldorf, Germany

INTRODUCTION: Pancreatic neuroendocrine tumors are rare and represent only about 1 to $2 \%$ of all neoplasias of the pancreas . They derive from hormone producing cells of the pancreas and are correspondingly designated as insulinomas, gastrinomas, VIPomas, glucogoninomas etc. The pathogenesis of this heterogenous family of tumors is largely unknown. RSUME was previously identified as sumoylation enhancer protein to stablize target genes such as HIF1a and IKBa. We found that RSUME is highly expressed in pancreas but loss of expression in PanNETs. Therefore the (patho-) physiological consequence due to RSUME absence was studied using PanNET derived BON1 cells. We found that RSUME knockdown in BON cells led to decrease HIF1 1 expression and vascular density and increased the liver metastasis tested in an orthotopic tumor model and the molecular mechanisms are partly attribute to decreased PTEN expression and increased NFKB activity.

\section{(1)}

\section{RSUME is down-regulated in PanNETs and regulates VEGF through HIF1a in PanNET cells}

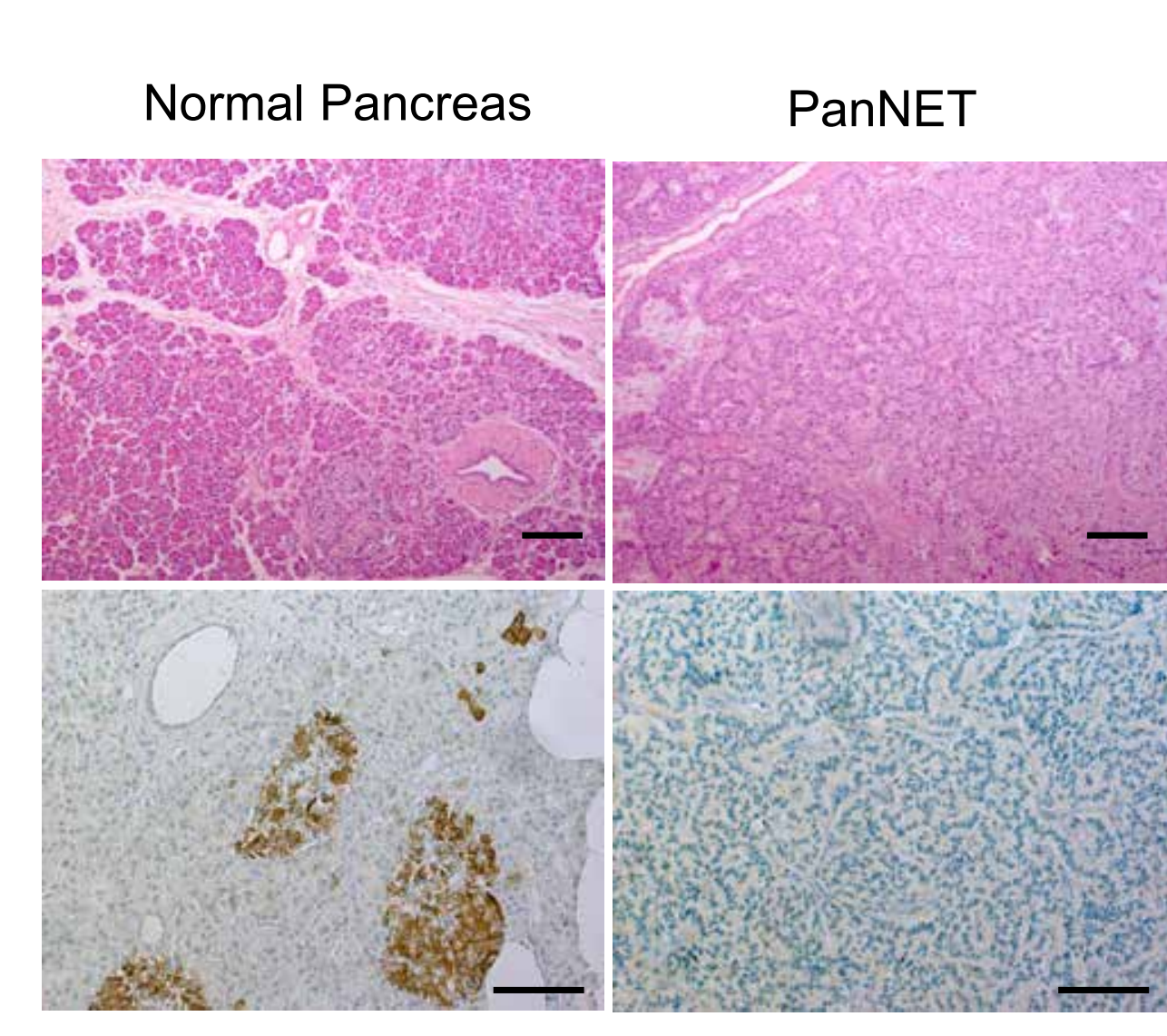

RSUME is downregualted in PanNETs
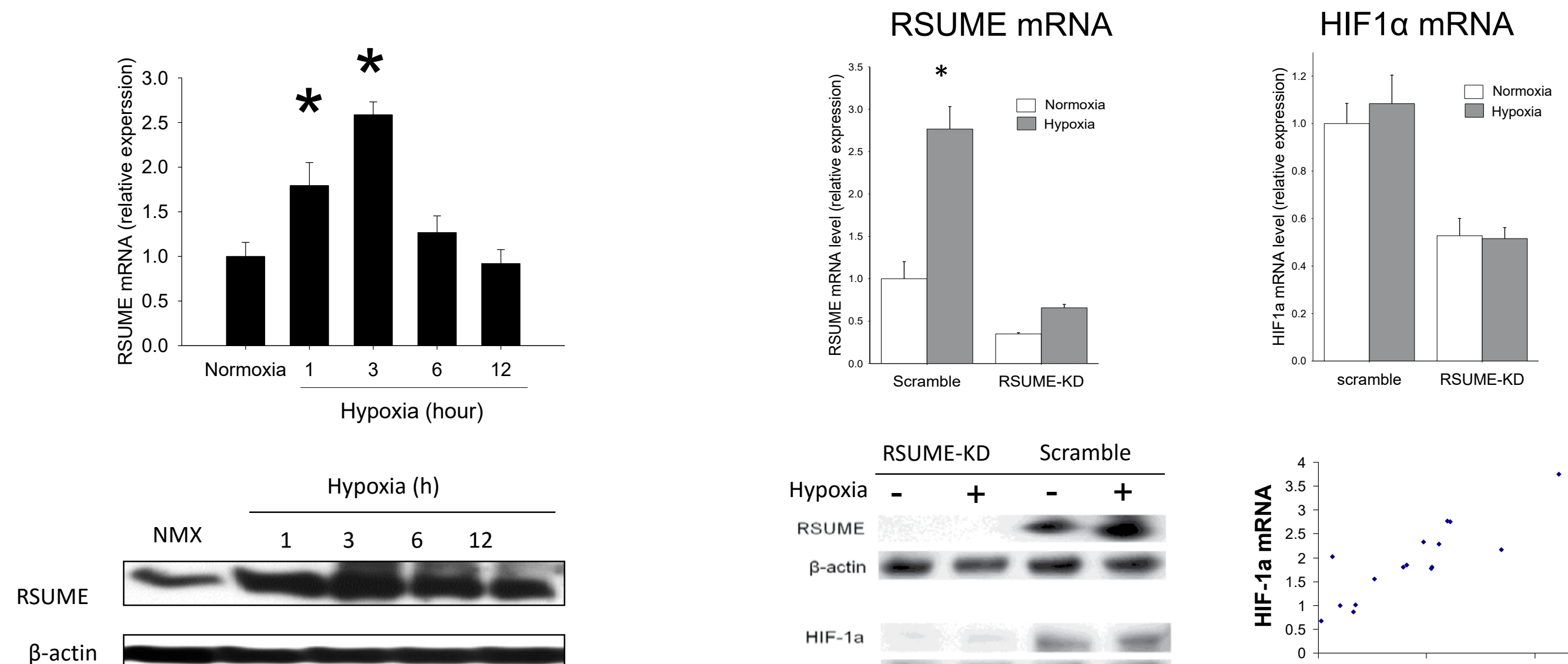

RSUME is induced during hypoxia

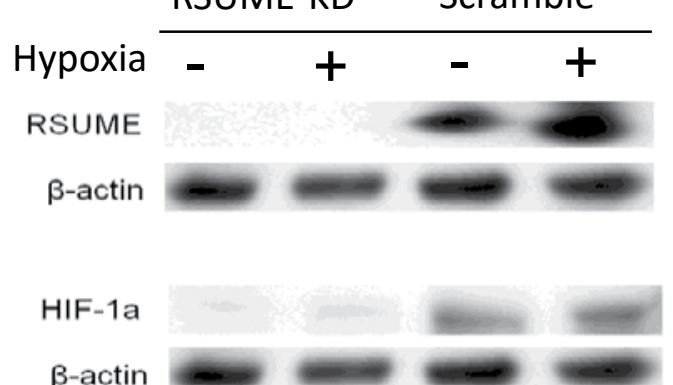

RSUME MRNA
mRNA ELISA

ELISA

(2)

RSUME negatively regulates NF-KB activity by enhancing IKBa SUMOylation

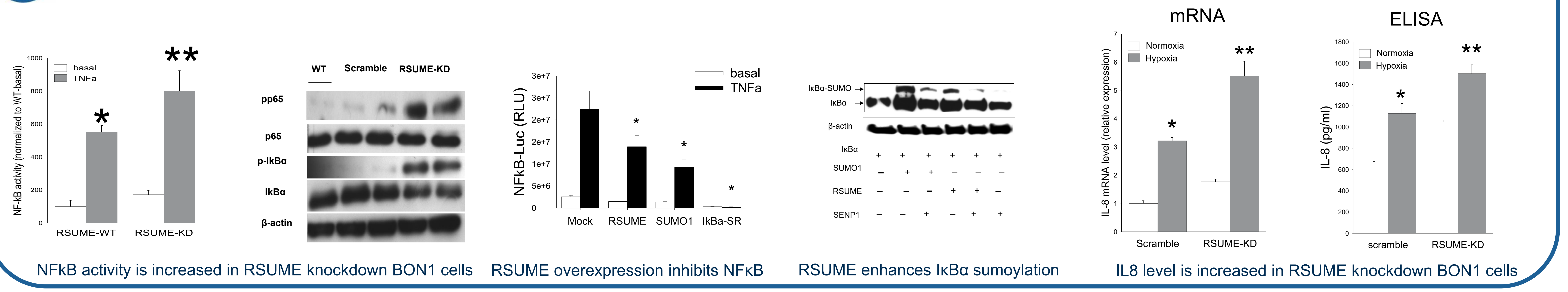

(3)

RSUME enhances PTEN sumoylation, protein stability and increases its nucleus accumulation

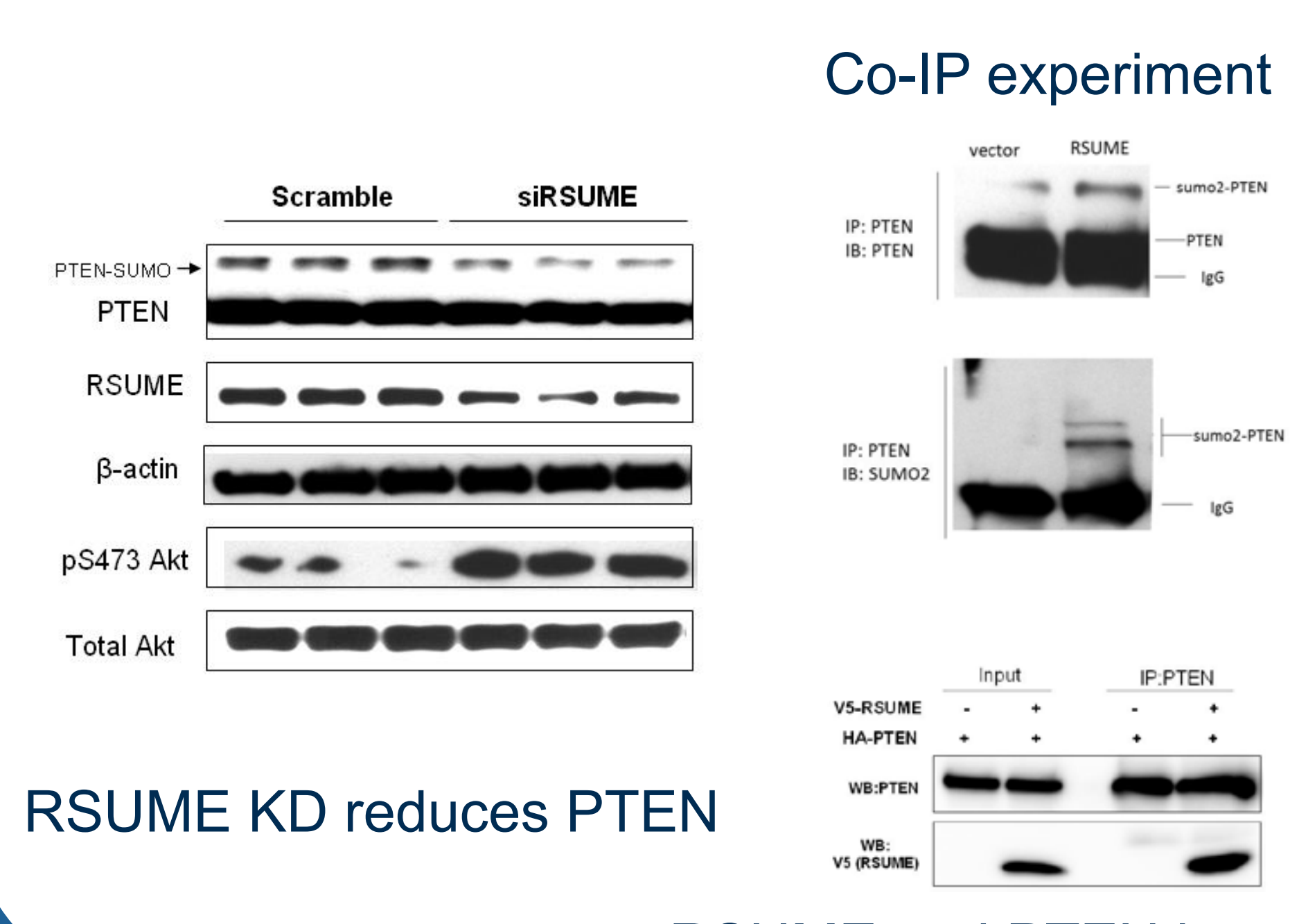

RSUME and PTEN interacts
In vivo sumoylation assay In vivo ubiquitination assay
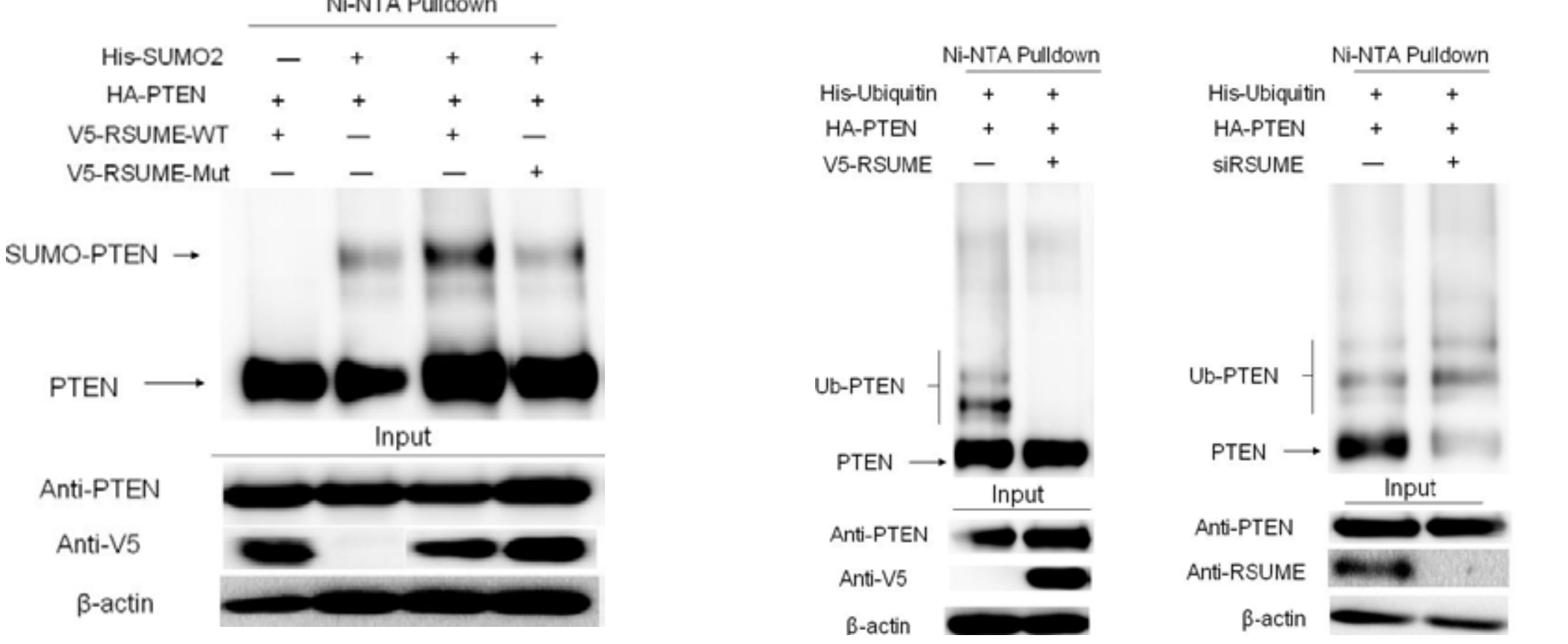

RSUME enhances PTEN increase protein stability
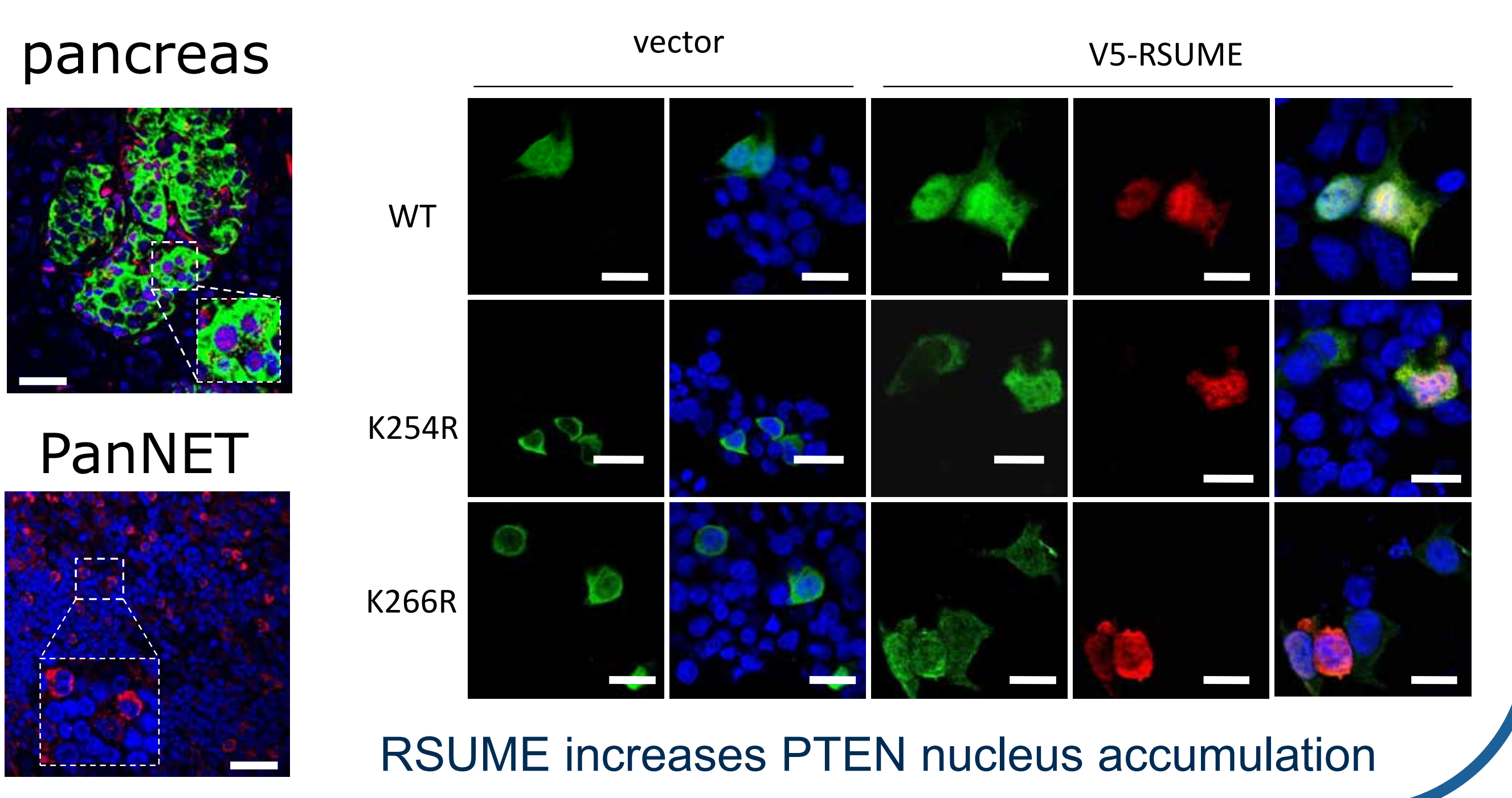

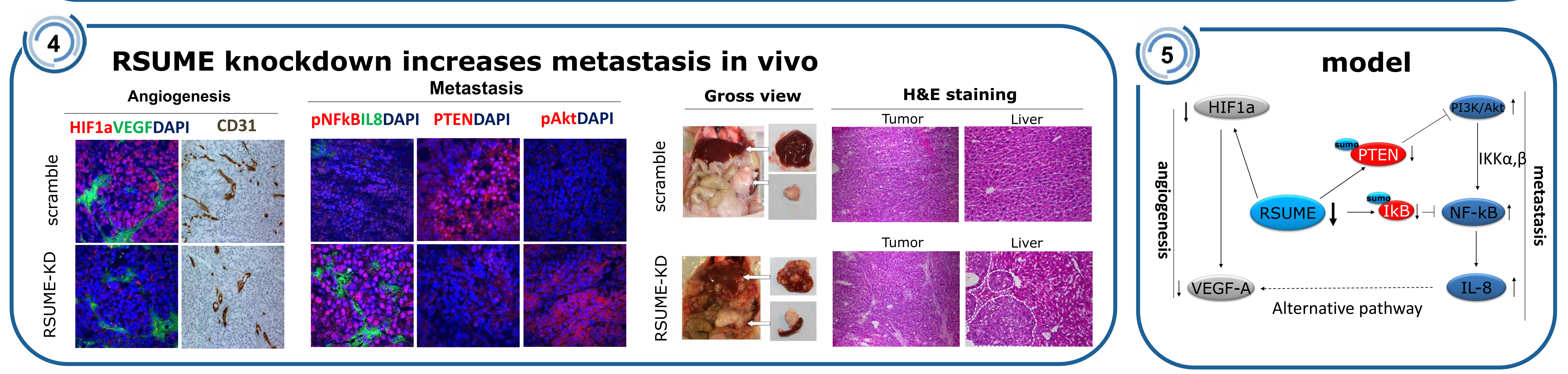

